\title{
Path Loss Model and Prediction of Range,
}

Frederic Heereman*, Wout Joseph, Emmeric Tanghe, David Plets, Leen Verloock and Luc Martens Department of Information Technology, Ghent University/IBBT

Gaston Crommenlaan 8 bus 201, B-9050 Ghent, Belgium

Tel.: +329331 4908; fax: +3293314899

*Email: frederic.heereman@intec.ugent.be

\begin{abstract}
In this paper, a path loss (PL) model for IEEE 802.11n in large conference rooms is proposed. The PL can be described accurately by a one-slope model with PL exponents varying from 1.2 to 1.7. The influence of humans (during a meeting) on the PL model is investigated. It was found that the PL exponent increases towards 2 in the presence of humans. Further, the effect of frequency $(2.4 / 5 \mathrm{GHz}$ ), antenna configuration (SISO vs MIMO $2 \times 2$ ), bandwidth (20 vs $40 \mathrm{MHz}$ ) and transmit power on the required number of access points for wireless conferencing, total radiated power consumption and maximum throughput is investigated. This is done by link budget calculation, based on our proposed PL model as well as the IEEE 802.11 TGn channel model. In this evaluation, it was found that the two PL models predict some essentially different effects concerning throughput and radiated power.
\end{abstract}

Keywords: Path loss; Conference room; IEEE 802.11n; Range; Power; Throughput

\section{INTRODUCTION}

The Wireless LAN Standard IEEE 802.11n, released in 2009, is an amendment to the previous standards 802.11a and $802.11 \mathrm{~g}$ to provide higher throughputs [1]. Modifications to the physical layer comprise MIMO (Multiple-Input Multiple-Output), the $2.4 / 5 \mathrm{GHz}$ band and a bandwidth of 20 or $40 \mathrm{MHz}$. Wireless conference systems (e.g. 24 audio and 2 video channels) may require throughputs of $60 \mathrm{Mbps}$ and more. 802.11n might be suitable for this application in large conference rooms.

In literature, both experimentally and theoretically determined propagation models have been reported for Wireless LAN in different indoor environments [2] - [9]. However, almost no path loss (PL) models can be found which are applicable for large conference rooms. The IEEE 802.11 TGn channel model could be applicable [3]. However, this model applies to very different types of environment (from residential to large space (indoors - outdoors)), and possibly does not take into account the specific geometry of large conference rooms (e.g. hemicycles).

In this paper, a PL model for large conference rooms is determined, based on PL measurements. This model will be compared with the TGn channel model. Further, the influence of humans (during a meeting) on the PL model will be characterized. Finally, we investigate experimentally whether our PL models accurately predict the received power of a real system. This is essential for range prediction by link budget calculation, which makes use of these PL models. 
Based on our proposed PL model, the effect of typical 802.11n features (including frequency, bandwidth and MIMO configuration) on the required number of access points, total power consumption (due to radiation) and

\section{Measurement CONFIgURATions AND SETUPS}

\section{A. Path loss measurement}

The path loss measurements were carried out in a large conference room in the European Parliament in Brussels. This room has a hemicycle geometry and contains about 350 seats (Fig. 1). The height of the ceiling is 7 to $8 \mathrm{~m}$. The measurements were done at frequencies 2.4 and $5.4 \mathrm{GHz}$, corresponding to the two bands of $802.11 \mathrm{n}$.

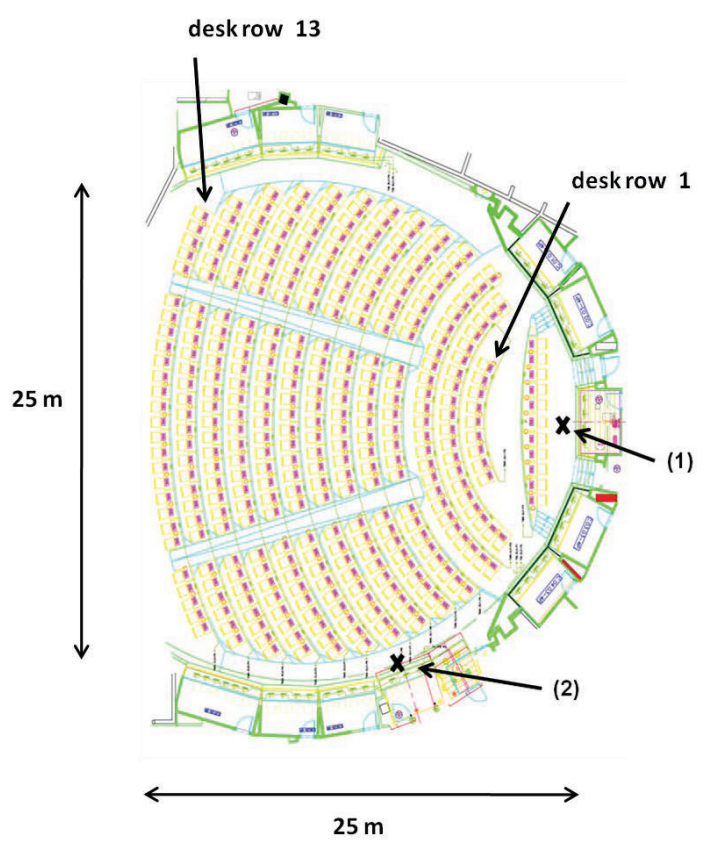

Fig. 1. Plan of a conference room in the European Parliament (Brussels), where PL measurements were carried out.

We considered two transmitter (Tx) positions. The first one is near the centre of the hemicycle ((1) in Fig. 1), at a height of $2 \mathrm{~m}$ and at a distance of $1 \mathrm{~m}$ from the wall. The second position is at the side of the room $((2)$ in Fig. 1), at a height of $3.5 \mathrm{~m}$ and about $10 \mathrm{~cm}$ from the wall. The Tx positions were chosen to obtain a line-of-sight condition for all the seats. The receiver $(\mathrm{Rx})$ was positioned just above the desks (i.e. the actual position of the clients). The measured trajectories, which the receiver moved along, included all rows of desks.

As measurement equipment at the Tx side, we used the Rohde \& Schwarz signal generator SMJ100A, connected to an omnidirectional transmitting antenna (type MAT-JAYBEAM MA431Z00 for $2.4 \mathrm{GHz}$ and European Antennas EVD2-5300/1285 for $5.4 \mathrm{GHz}$ ). The equipment at the Rx side included a receiving antenna (of the same type as Tx), connected to the Hewlett Packard spectrum analyzer 8561B, and a tachometer. The spectrum analyzer and the 
tachometer were connected to a laptop, which saved the received power and the distance along the Rx trajectory as a function of time.

During the measurements, no people were present in the room, because there was no permission to execute measurements during meetings in the European Parliament. Consequently, our measurements allow to determine large-scale spatial fading, but no temporal fading. In addition, the presence of people can have an influence on the developed PL model.

\section{B. PL measurement in a university auditorium}

To investigate the influence of humans on the PL model, PL measurements were carried out in a university auditorium during a lecture and repeated in the absence of people. The area of this auditorium (see Fig. 2) is $12 \mathrm{~m} \times 20 \mathrm{~m}$ and the height of the ceiling is $4.6 \mathrm{~m}$. There are 17 desk rows of 16 seats. During the lecture, about 80 people were present. The occupation of the seats was nearly homogeneous. The measurement equipment and the frequencies were the same as for the conference room in the European Parliament (Section II-A). Tx was positioned in front of the auditorium (in the corner, $1.7 \mathrm{~m}$ from the walls) at a height of $2.2 \mathrm{~m}$ (Fig. 2). The height of Rx was $1.2 \mathrm{~m}$ (i.e. above the desks). The measured $\mathrm{Rx}$ trajectories were row 8, 11, 13 and 16 (Fig. 2). These rows were left unoccupied to provide passage of the measurement equipment.

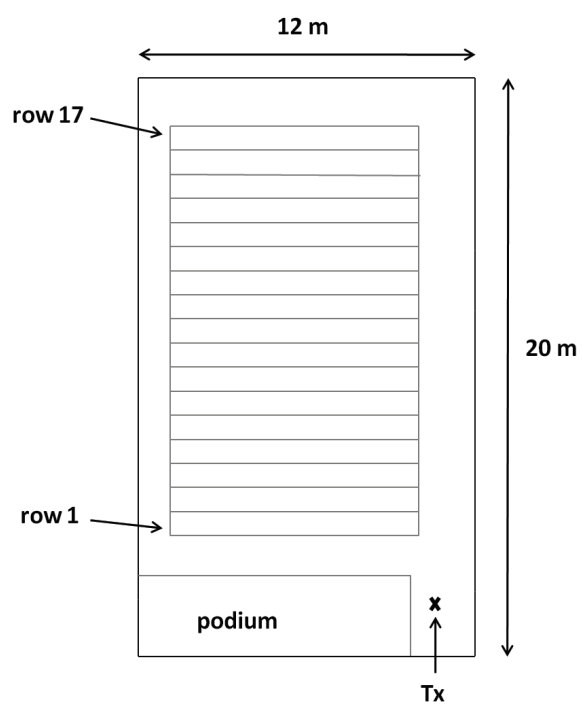

Fig. 2. Plan of a university auditorium, where PL measurements were carried out.

\section{Measurement with a 802.11n system}

To investigate whether our PL models predict accurately the received power of different equipment, we have set up an actual 802.11n system with $2 \times 2$ MIMO configuration in the large conference room (European Parliament). Broadcast packets were generated on an access point with Atheros 802.11n chipset (type 9220/9280 Merlin). At the Rx side, the equipment included a laptop, equipped with a Wireless USB Adapter (Proxim ORiNOCO 802.11a/b/g/n USB Adapter) and Airmagnet WiFi Analyzer PRO software [10], as well as a tachometer, connected to the laptop. All this equipment was set on a cart, which was moved along all rows of desks. The received signal strength, 
measured by Airmagnet, and the distance (along the trajectory), measured by the tachometer, were registered by the laptop as a function of time.

The measurements were done in the $2.4 \mathrm{GHz}$ band (channel 6) and the $5 \mathrm{GHz}$ band (channel 44). The following link parameters were used: $20 \mathrm{MHz}$ bandwidth, Modulation \& Coding Scheme (MCS) 0 (i.e. modulation BPSK 1/2), a transmit power of $17 \mathrm{dBm}$ and a packet size of 1000 bytes payload. The parameters (bandwidth, MCS and transmit power) were chosen to obtain coverage in the whole room.

The access point was positioned in front of the room, again at a height of $2 \mathrm{~m}$ and at a distance of $1 \mathrm{~m}$ from the wall (Fig. 1). The receiver was positioned again just above the desks, and moved along all rows of desks.

\section{PATH LOSS MODEL}

\section{A. Development of path loss model}

From the measurement data, the path loss $[\mathrm{dB}]$ is calculated by

$$
P L=-\left\langle P_{R}\right\rangle+P_{T}+G_{T}+G_{R}-L_{T}-L_{R},
$$

where $\left\langle P_{R}\right\rangle$ is the averaged received power $\left(\mathrm{P}_{\mathrm{R}}\right)[\mathrm{dBm}], \mathrm{P}_{\mathrm{T}}$ is the transmit power $[\mathrm{dBm}], \mathrm{G}_{\mathrm{T}}\left(\mathrm{G}_{\mathrm{R}}\right)$ is the transmitter (receiver) gain $[\mathrm{dBi}]$, and $\mathrm{L}_{\mathrm{T}}\left(\mathrm{L}_{\mathrm{R}}\right)$ is the transmitter (receiver) feeder loss $[\mathrm{dB}]$.

From the measurement data, the $\mathrm{P}_{\mathrm{R}}$ samples and their corresponding position (distance along measured trajectory) are obtained. To calculate $\left\langle P_{R}\right\rangle$, the $\mathrm{P}_{\mathrm{R}}$ samples are averaged over a distance of $10 \lambda$, where $\lambda$ is the wavelength, to cancel out small-scale fading [9].

During the measurements, a transmit power of $15 \mathrm{dBm}$ is used. The losses of the cables (with a length of $8 \mathrm{~m}$ and $4 \mathrm{~m}$ resp.) are determined experimentally: $\mathrm{L}_{\mathrm{T}}$ is $4.1 \mathrm{~dB}$ at $2.4 \mathrm{GHz}$ and $7.6 \mathrm{~dB}$ at $5.4 \mathrm{GHz} ; \mathrm{L}_{\mathrm{R}}$ is $2.2 \mathrm{~dB}$ at $2.4 \mathrm{GHz}$ and $3.5 \mathrm{~dB}$ at $5.4 \mathrm{GHz}$.

We determine the gain $(\mathrm{G})$ of transmitter and receiver as follows:

$$
G=G_{\max }+F(\theta),
$$

where $\mathrm{G}_{\max }$ is the (maximal) gain $\left[\mathrm{dBi}\right.$ ] in the horizontal plane, and $\mathrm{F}$, defined by $\mathrm{G}-\mathrm{G}_{\max }$, depends on the elevation angle $\theta$. It is necessary to consider an angle-dependent gain, since angles $\theta$ (between $\mathrm{Tx}$ and $\mathrm{Rx}$ ) up to $47^{\circ}$ are present (in the conference room in the European Parliament), and the $3 \mathrm{~dB}$ beamwidth is $40^{\circ}$ and $80^{\circ}$ for the $2.4 \mathrm{GHz}$ and $5.4 \mathrm{GHz}$ antenna respectively. For the antennas used at $2.4 \mathrm{GHz}$, we use $\mathrm{G}_{\max }$ and $\mathrm{F}(\theta)$ from the datasheet of the manufacturer. For the antennas used at $5.4 \mathrm{GHz}$, we know $\mathrm{G}_{\max }$ from the datasheet, but have no data for $\mathrm{F}(\theta)$. Therefore, we determine $\mathrm{F}$ by a theoretical approximation, applying to thin wire dipole antennas, proposed in [11]:

$$
F=10 \log \left(\left(\frac{\cos (k L \sin (\theta))-\cos (k L)}{\cos (\theta)(1-\cos (k L))}\right)^{2}\right),
$$

where $\mathrm{k}=2 \pi / \lambda$, and $2 \mathrm{~L}$ is the length of the antenna. The $3 \mathrm{~dB}$ beamwidth (available in the datasheet) allows to determine the parameter $\mathrm{kL}$ in (3): $\mathrm{kL}=1.426$.

The PL samples are calculated with (1), in positions (along the trajectory) with a separation of $\lambda / 40$ (i.e. a moving average). We determine PL models for the large conference room (European Parliament) for the different cases (2 frequencies, 2 Tx positions). 


\section{B. Path loss model: results and discussion}

For the large conference room (European Parliament), we describe the path loss $[\mathrm{dB}]$ versus distance $\mathrm{d}[\mathrm{m}]$ between Tx and Rx by a one-slope model, with one standard deviation $\sigma$ [dB]:

$$
P L=P L_{0}+10 n \log (d)+X_{\sigma}
$$

where $\mathrm{PL}_{0}$ is the mean path loss at a distance of $1 \mathrm{~m}, \mathrm{n}$ is the PL exponent and $\mathrm{X}_{\sigma}[\mathrm{dB}]$ is a normally distributed variable with $0 \mathrm{~dB}$ median and standard deviation $\sigma$. The parameters $\mathrm{PL}_{0}, \mathrm{n}$ and $\sigma$, determined by the method of least squares, are shown in Table I, as well as the Tx-Rx separation where the PL could be experimentally determined. The determined PL exponents vary from 1.2 to 1.7 , which is lower than the free-space PL exponent of 2. This can be explained by reflections at the walls, the ceiling and the floor. This gives an additional, rather homogeneous contribution to the free-space received power, which lowers the PL exponent. The same explanation was given in [12], where PL exponents of 1.5 to 1.7 were reported for a class room. Also in [9], PL exponents of 1.3 to 1.7 were found in factory buildings for wood and metal processing (in line-of-sight condition) and attributed to multipath propagation.

TABLE I

PARAMETERS OF PL MODEL, BASED ON PL MEASUREMENTS IN A LARGE CONFERENCE ROOM.

\begin{tabular}{|c|c|c|c|c|c|c|}
\hline frequency & $\begin{array}{c}\mathrm{Tx} \\
\text { position }\end{array}$ & $\mathrm{n}$ & $\begin{array}{c}\mathrm{PL}_{0} \\
{[\mathrm{~dB}]}\end{array}$ & $\begin{array}{c}\mathrm{d}_{\mathrm{br}} \\
{[\mathrm{m}]}\end{array}$ & $\begin{array}{c}\sigma \\
{[\mathrm{dB}]}\end{array}$ & $\begin{array}{c}\text { considered } \\
\text { Tx-Rx separation }\end{array}$ \\
\hline \multirow{2}{*}{$2.4 \mathrm{GHz}$} & front & 1.4 & 43 & 3.9 & 2.0 & $5-24 \mathrm{~m}$ \\
\cline { 2 - 7 } & side & 1.7 & 40 & 1.2 & 2.2 & $5-26 \mathrm{~m}$ \\
\cline { 2 - 7 } & all & 1.6 & 42 & 2.5 & 2.1 & \\
\hline \multirow{2}{*}{$5.4 \mathrm{GHz}$} & front & 1.2 & 51 & 3.0 & 2.3 & $5-24 \mathrm{~m}$ \\
\cline { 2 - 7 } & side & 1.2 & 53 & 4.9 & 2.2 & $5-27 \mathrm{~m}$ \\
\cline { 2 - 7 } & all & 1.2 & 52 & 3.9 & 2.4 & \\
\hline
\end{tabular}

For all cases, we found that it is possible to describe the path loss accurately by a one-slope model with a standard deviation of about $2 \mathrm{~dB}$. This is illustrated in Fig. 3, where percentiles, based on PL samples from a local region of $4 \mathrm{~m}$, are shown. The median can be modeled by a one-slope model, with a deviation less than $1 \mathrm{~dB}$. The shift between the 75th percentile and the median is almost constant, which indicates a single standard deviation.

Assuming that, for short Tx-Rx separation, the dominant path arriving at Rx is the line-of-sight path, the median of the PL samples should equal the free-space PL for short Tx-Rx separation (as proposed in the TGn model [3]). In this case, it is useful to express the PL model with a breakpoint [3]:

$$
P L=P L_{f r e e, 0}+10 n \log \left(d / d_{b r}\right)+X_{\sigma},
$$

where breakpoint $\mathrm{d}_{\mathrm{br}}$ is the distance $[\mathrm{m}]$ between $\mathrm{Tx}$ and $\mathrm{Rx}$ where the one-slope model intersects with the free-space path loss, and $\mathrm{PL}_{\text {free, } 0}$ is the free-space path loss $[\mathrm{dB}]$ at distance $\mathrm{d}_{\mathrm{br}}$. The corresponding breakpoint parameters, shown in Table I, vary from 1 to $5 \mathrm{~m}$. These breakpoints are smaller than the minimal considered Tx-Rx separation of $5 \mathrm{~m}$ (see Table I), in agreement with the fact that the PL samples can be accurately modeled by a one-slope model. 


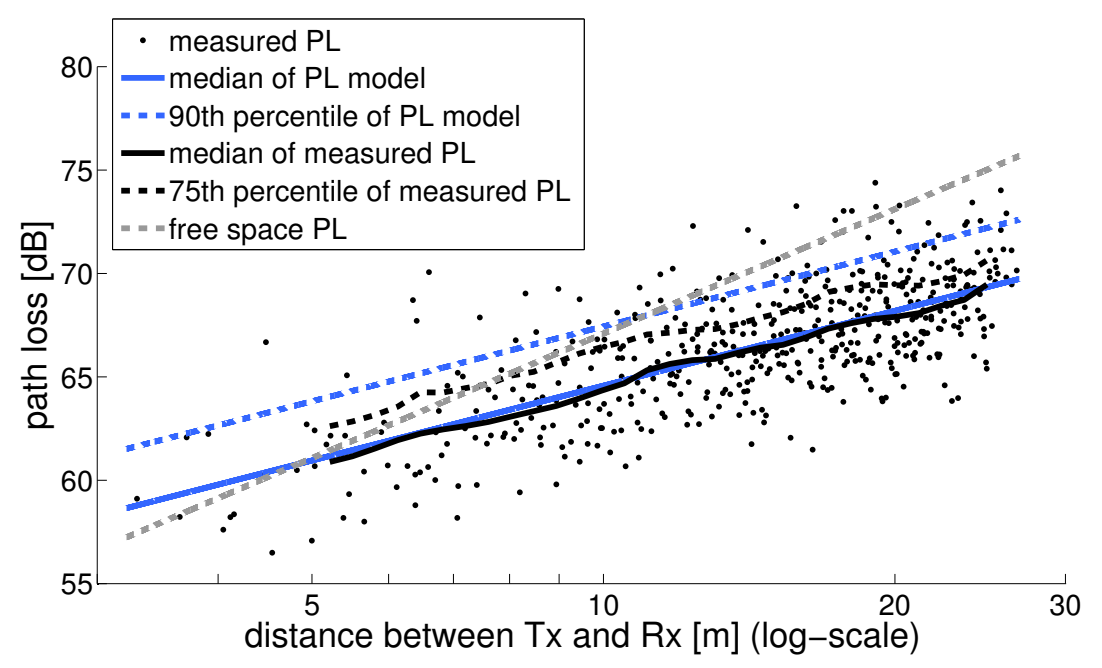

Fig. 3. Measured PL and PL model in a large conference room (at $5.4 \mathrm{GHz}$, Tx position at the side). Percentiles based on the measured PL samples show that the PL can be described accurately by a one-slope model with a single standard deviation. For clarity, only PL samples of positions separated by $10 \lambda$ are shown.

The expression using a breakpoint (see (5)) gives more insight in the frequency dependency of the PL model than the expression with an intercept $\mathrm{PL}_{0}$. Firstly, the PL is proportional to $1 / \lambda^{2}$ (where $\lambda$ is the wavelength) via the term $\mathrm{PL}_{\text {free, } 0}$ (first contribution $\mathrm{C}_{1}$ ). This is due to the interaction between electromagnetic field and antenna. Secondly, the frequency has also an influence on the interaction between electromagnetic field and environment, which is reflected in $\mathrm{n}$ (attenuation), $\mathrm{d}_{\mathrm{br}}$ (transition from free-space PL to different attenuation mechanism) and $\sigma$ (large-scale fading) (second contribution $\mathrm{C}_{2}$ ). The influence of the frequency on $\mathrm{n}, \mathrm{d}_{\mathrm{br}}$ and $\sigma$ can be seen in Table $\mathrm{I}$. The two effects of the frequency on the PL have been compared by calculating the ratio $\mathrm{R}$ of the first and the second contribution to the PL:

$$
R=\frac{C_{1,5.4 \mathrm{GHz}}-C_{1,2.4 \mathrm{GHz}}}{C_{2,5.4 \mathrm{GHz}}-C_{2,2.4 \mathrm{GHz}}}=\frac{20 \log (5.4 \mathrm{GHz} / 2.4 \mathrm{GHz})}{\left|P L_{5.4 \mathrm{GHz}}(d)-P L_{2.4 \mathrm{GHz}}(d)-20 \log (5.4 \mathrm{GHz} / 2.4 \mathrm{GHz})\right|},
$$

where $\mathrm{PL}_{5.4 \mathrm{GHz}}(\mathrm{d})$ and $\mathrm{PL}_{2.4 \mathrm{GHz}}(\mathrm{d})$ are the 90 th percentile $\mathrm{PL}[\mathrm{dB}]$ at $5.4 \mathrm{GHz}$ and $2.4 \mathrm{GHz}$ resp., calculated with our PL models (Table I) at Tx-Rx separation d. This ratio R is found to be minimal 2.7 for Tx in front of the room, and minimal 3.8 for Tx on the side. This shows that the first contribution (PL proportional to $1 / \lambda^{2}$ ) is stronger than the second one. This explains that the $\mathrm{PL}_{0}$ values (listed in Table I) are higher at $5.4 \mathrm{GHz}$ than at $2.4 \mathrm{GHz}$.

According to the IEEE 802.11 TGn channel model [3], the PL can be modeled by the free-space PL for $\mathrm{d}<\mathrm{d}_{\mathrm{br}}$, and by a one-slope model with exponent 3.5 for $d>d_{b r}$. The TGn model predicts a breakpoint of $20 \mathrm{~m}$ for 'Large office' (type of environment 'E') and $30 \mathrm{~m}$ for 'Large space (indoors - outdoors)' (type of environment 'F'). Compared to the TGn channel model, the PL model, proposed here, has a lower breakpoint and a lower PL exponent for $d>d_{b r}$. This results in much lower PL values. This is illustrated in Fig. 4, where the PL models (PL median), developed for conference rooms, and the TGn PL models for types of environment ' $\mathrm{E}$ ' and ' $\mathrm{F}$ ' are shown for $2.4 \mathrm{GHz}$. The PL median of our proposed models is up to $6 \mathrm{~dB}$ and $8 \mathrm{~dB}$ lower than the PL median of the TGn model at $2.4 \mathrm{GHz}$ and $5.4 \mathrm{GHz}$ respectively. The range predicted by our PL model is up to a factor 1.6 higher than the range predicted by the TGn model at $2.4 \mathrm{GHz}$ (see Fig. 4) and a factor 2.3 higher at $5.4 \mathrm{GHz}$. Assuming that the required number of access points (\#AP) is inversely proportional to the square of the range, \#AP decreases with a factor 2.7 and 5.1 respectively according to our PL model. We conclude that our PL model is significantly 
better than the TGn model for range prediction.

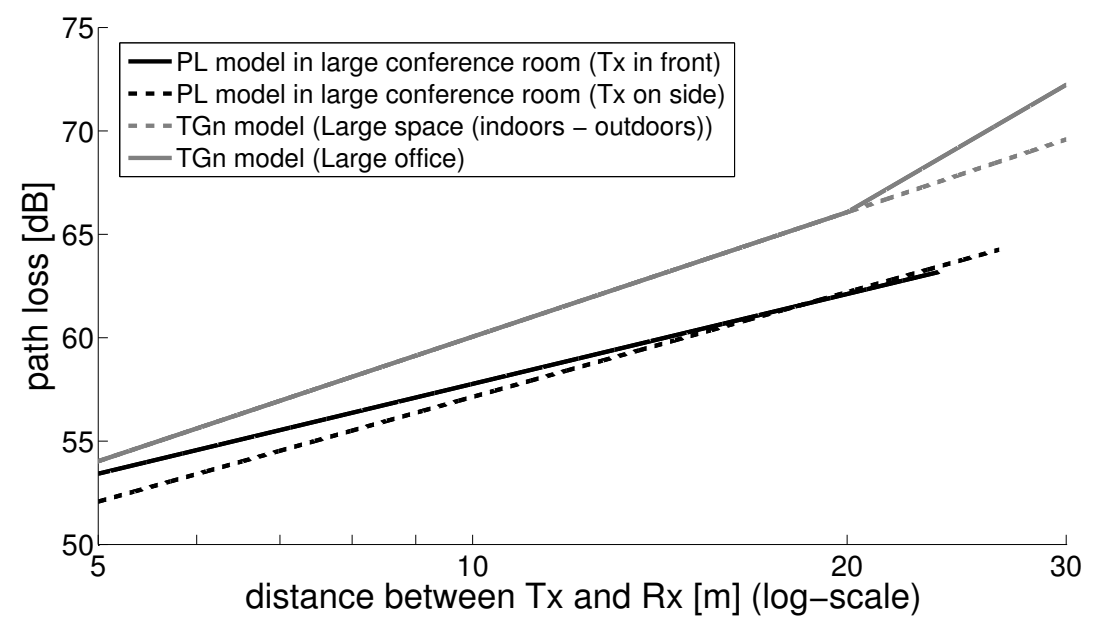

Fig. 4. Comparison of our PL models (PL median), developed for conference rooms, with the TGn PL models for 'Large office' and 'Large space (indoors - outdoors)' at $2.4 \mathrm{GHz}$.

\section{Influence of humans on the PL model}

Based on the PL measurement in the university auditorium, one-slope PL models are obtained in the same way as described in Section III-A. In Table II, the parameters of these PL models are listed for the case of the empty room and the room with a lecture at $2.4 \mathrm{GHz}$ and $5.4 \mathrm{GHz}$. Fig. 5 shows the median of these PL models. At $2.4 \mathrm{GHz}$, the PL exponent $\mathrm{n}$ during the lecture is almost a factor 2 higher than in the empty room (Table II) and the intercept $\mathrm{PL}_{0}$ is about $8 \mathrm{~dB}$ lower. The latter is due to a higher PL exponent, while the PL during the lecture intersects with the PL in the empty room at a Tx-Rx separation of about $10 \mathrm{~m}$ (see Fig. 5). At $5.4 \mathrm{GHz}$, the deviation of the PL median between the lecture and the empty room is smaller than $0.5 \mathrm{~dB}$ (Fig. 5), which is negligible for range prediction. At both $2.4 \mathrm{GHz}$ and $5.4 \mathrm{GHz}$, the difference of the standard deviation $\sigma$ between the lecture and the empty room is also negligible $(<0.2 \mathrm{~dB})$ (Table II).

These findings can be explained as follows. At $2.4 \mathrm{GHz}$, the PL exponent $\mathrm{n}$ in the empty room is lower than the free-space PL exponent (i.e. 2) due to multipath propagation (as mentioned before). In the presence of humans, the PL exponent increases towards 2 due to the absorption by the human body (see Table II). This occurs from a Tx-Rx separation of about $10 \mathrm{~m}$ (Fig. 5). This might be related to the Tx-Rx separation at which the crowded area starts. During the lecture, the minimal distance between Tx and the humans was about $4 \mathrm{~m}$. At $5.4 \mathrm{GHz}$, the PL exponent in the empty room is about 2 (Table II), which is almost the same during the lecture.

For the large conference room in the European Parliament, we similarly expect that the attenuation increases in the crowded area, which results in a higher PL exponent (towards 2). Consequently, the intercept $\mathrm{PL}_{0}$ will decrease in the presence of humans, since the minimal distance between Tx and the humans is larger than $1 \mathrm{~m}$. 
TABLE II

PARAMETERS OF PL MODEL IN A UNIVERSITY AUDITORIUM IN THE ABSENCE OF HUMANS AND DURING A LECTURE AT 2.4 GHz AND $5.4 \mathrm{GHz}$.

\begin{tabular}{|c|c|c|c|c|}
\cline { 3 - 5 } \multicolumn{2}{c|}{} & $\mathrm{n}$ & $\mathrm{PL}_{0}[\mathrm{~dB}]$ & $\sigma[\mathrm{dB}]$ \\
\hline \multirow{2}{*}{$2.4 \mathrm{GHz}$} & empty room & 0.99 & 47.7 & 0.94 \\
\cline { 2 - 5 } & lecture & 1.76 & 39.8 & 0.91 \\
\hline \multirow{2}{*}{$5.4 \mathrm{GHz}$} & empty room & 2.04 & 43.4 & 1.15 \\
\cline { 2 - 5 } & lecture & 1.88 & 45.4 & 1.32 \\
\hline
\end{tabular}

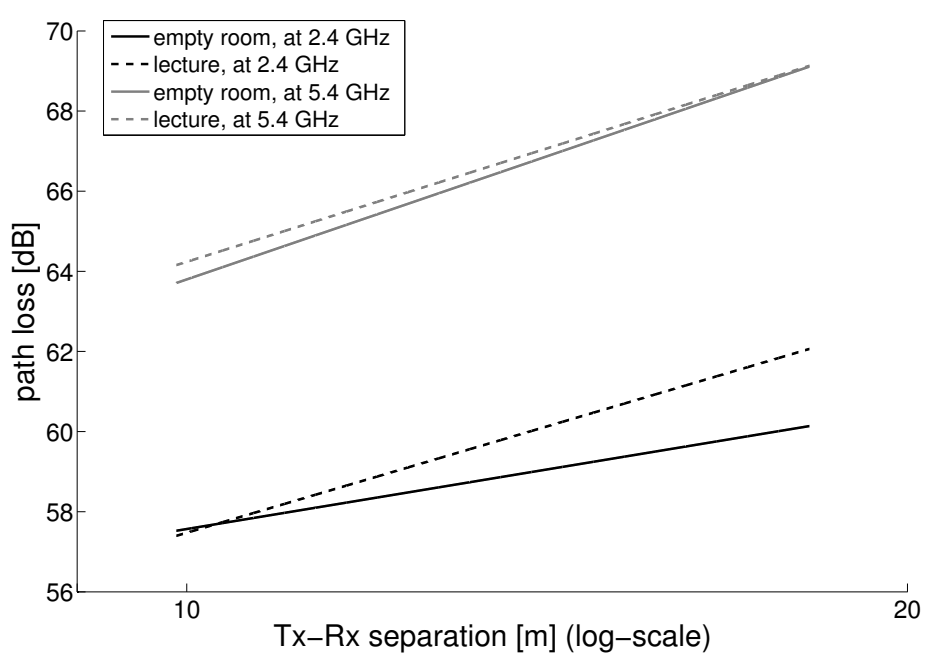

Fig. 5. PL model in a university auditorium in the absence of humans and during a lecture at $2.4 \mathrm{GHz}$ and $5.4 \mathrm{GHz}$.

\section{ApPliCABILITy OF THE PL MODEL TO A REAL SYSTEM}

In this section, we investigate how accurately our proposed PL model can predict the received power of a real 802.11n system. This is essential for range prediction from link budget calculation. Therefore, we carried out a measurement with a Wireless USB Adapter and Airmagnet software (as described in Section II-C) in the same room as the PL measurement (the conference room in the European Parliament). Based on the received power, registered by Airmagnet, the PL should be calculated with (1) and compared with the PL of the PL measurement. The PL of the two measurements should be equal, which means that our PL models can predict accurately the received power for other systems. However, not all required specifications (antenna gain, radiation pattern or beamwidth, cable loss) could be obtained from the datasheets and manufacturers of the access point and Wireless USB Adapter. Consequently, the PL for the real system is known, apart from a shift. Actually, this is only true for desk rows at a higher distance from Tx. For desk rows which are closer to Tx, the elevation angle between $\mathrm{Tx}$ and $\mathrm{Rx}$ is no longer small compared to the antenna beamwidth, and the gains in (1) become dependent on the Tx-Rx separation. For the first and the last desk row, the elevation angle between $\mathrm{Tx}$ and $\mathrm{Rx}$ is $23^{\circ}$ and $2^{\circ}$ respectively.

Per desk row, the median and the 75th percentile of the PL samples are calculated for the PL measurement and for the measurement with the Wireless USB Adapter. To compare the PL of both measurements, we shift the PL of the measurement with the Wireless USB Adapter, in order that the PL median values fit to the PL median values of the PL measurement. Here, only desk rows 6 to 13 are considered. Indeed, for these rows, the elevation angle 
between $\mathrm{Tx}$ and $\mathrm{Rx}$ is small $\left(<7^{\circ}\right)$, so that we can expect that the gains in (1) do not vary much $(<0.5 \mathrm{~dB})$ with the Tx-Rx separation.

The comparison of the PL from the PL measurement with the (shifted) PL from the measurement with the Wireless USB Adapter is illustrated in Fig. 6 for channel $6(2.4 \mathrm{GHz})$. Here, the PL median and 75th percentile for each desk row are shown for both measurements. This comparison gives an idea of (i) the minimal deviation (between the PL measurement and the measurement with the Wireless USB Adapter) of the PL median and (ii) the deviation of the PL standard deviation. These deviations are summarized in Table III for channel $6(2.4 \mathrm{GHz})$ and $44(5.4 \mathrm{GHz})$. Here, the average and maximum deviation of the PL median is based on desk rows 6 to 13 only, because the radiation pattern of the antennas could not be taken into account, which is important for smaller Tx-Rx separation (as mentioned before). The deviation (average and maximum value) of the PL standard deviation is based on all desk rows, because a PL standard deviation for a certain desk row concerns the variation of PL at the same Tx-Rx separation. As can be seen in Table III, a deviation of the PL median of about $1 \mathrm{~dB}$ can occur. Since the median PL values have been fitted, it cannot be excluded that higher deviations of the PL median are possible. Concerning the deviation of the PL standard deviation, deviations of about $2 \mathrm{~dB}$ can occur (Table III). These deviations ( 1 or $2 \mathrm{~dB}$ ) fall within the measurement uncertainty of the Wireless USB Adapter. Deviations can also be caused by the use of different equipment (e.g. different radiation pattern). Indeed, the measured PL differs from the free-space PL (as discussed before), which indicates multipath propagation.

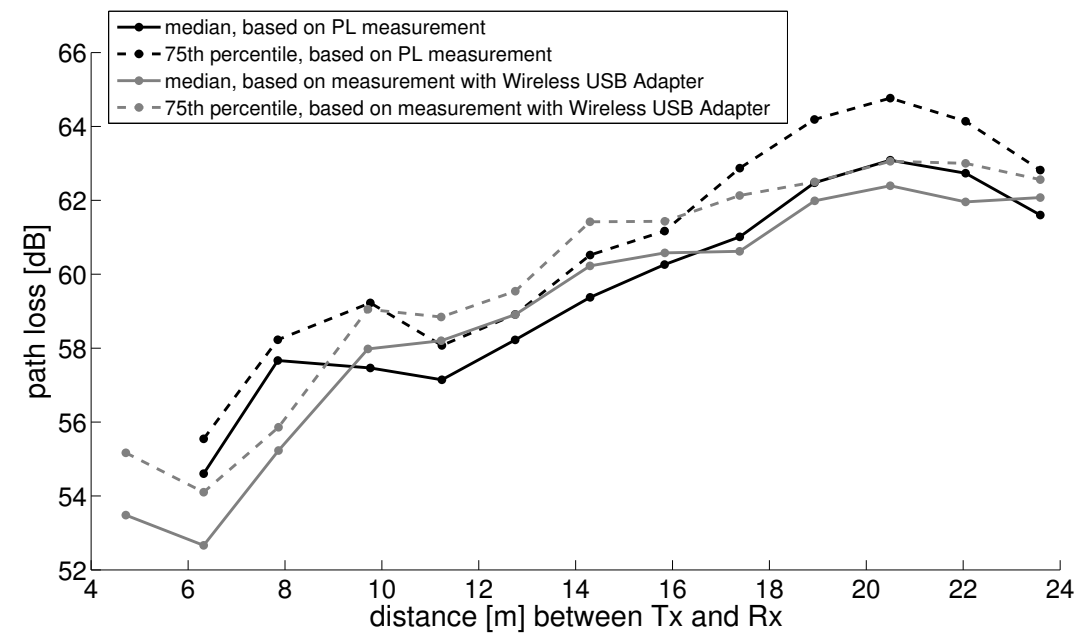

Fig. 6. Comparison of PL from the PL measurement with PL from the measurement with the Wireless USB Adapter (channel 6 (2.4 GHz)).

TABLE III

COMPARISON OF PL FROM THE PL MEASUREMENT WITH PL FROM THE MEASUREMENT WITH THE WIRELESS USB ADAPTER: THE DEVIATION (AVERAGE AND MAXIMUM VALUE) OF THE PL MEDIAN AND OF THE PL STANDARD DEVIATION.

\begin{tabular}{|c|c|c|c|c|}
\cline { 2 - 5 } \multicolumn{1}{c|}{} & \multicolumn{2}{c|}{ channel $6(2.4 \mathrm{GHz})$} & \multicolumn{2}{c|}{ channel $44(5 \mathrm{GHz})$} \\
\cline { 2 - 5 } & avg deviation & max deviation & avg deviation & max deviation \\
\hline PL median $[\mathrm{dB}]$ & 0.6 & 0.9 & 0.5 & 1.2 \\
\hline PL standard deviation $[\mathrm{dB}]$ & 0.7 & 1.8 & 0.9 & 1.8 \\
\hline
\end{tabular}




\section{RANGE, POWER CONSUMPTION AND THROUGHPUT OF 802.11N IN LARGE CONFERENCE ROOMS}

\section{A. Calculation of the range, number of access points and power consumption}

In this section, an evaluation of the following parameters is made, with the focus on large conference rooms: SISO vs MIMO 2×2:1 / MIMO 2×2:2 (i.e. 1 and 2 spatial streams resp.), $2.4 \mathrm{GHz}$ vs $5 \mathrm{GHz}$ band, 20 vs $40 \mathrm{MHz}$ bandwidth and transmit power. In this evaluation, the required number of access points (\#AP), the required total radiated power consumption $(\mathrm{P})$ and the maximum possible (physical) throughput $\left(\mathrm{TP}_{\max }\right)$ are considered. The evaluation is based on the TGn model as well as our proposed PL model, which allows to thoroughly compare the two models. It is assumed that the access points are positioned according to a fixed range, which is achieved by tuning the transmit power. We consider a Guard Interval of $800 \mathrm{~ns}$. The data rate varies from 6.5 Mbps (BPSK 1/2) to $65 \mathrm{Mbps}$ (64-QAM 5/6) for SISO or MIMO 2×2:1 and from 13 Mbps (BPSK 1/2) to 130 Mbps (64-QAM 5/6) for MIMO 2×2:2 [13].

The evaluation is based on the calculation of the range $\mathrm{R}[\mathrm{m}]$, using the link budget relation [10]:

$$
P_{T}-P_{R}+G_{T}+G_{R}-L_{T}-L_{R}=P L_{0}+10 n \log (R)+M_{S}+M_{F},
$$

where $P_{R}$ is the receiver sensitivity $[\mathrm{dBm}], \mathrm{M}_{S}[\mathrm{~dB}]$ is the shadowing margin and $\mathrm{M}_{F}[\mathrm{~dB}]$ is the temporal fading margin. The required number of access points (\#AP) is then calculated as

$$
\# A P=S /\left(\pi R^{2}\right)
$$

where $\mathrm{S}\left[\mathrm{m}^{2}\right]$ is the area of the room. The required total power consumption $\mathrm{P}[\mathrm{mW}]$, due to radiation, is calculated as

$$
P=\# A P P_{T} .
$$

For the calculations, receiver sensitivities of an 802.11n 'reference' receiver are used [1]. Compared to SISO, the sensitivities are decreased by $6 \mathrm{~dB}$ for MIMO $2 \times 2: 1$ (due to diversity gain) and are assumed to be the same for MIMO $2 \times 2: 2$ [14]. Compared to a bandwidth of $20 \mathrm{MHz}$, the sensitivities are increased by $3 \mathrm{~dB}$ for $40 \mathrm{MHz}$.

The calculation is done for $G_{T}=G_{R}=2 \mathrm{dBi}$ and $L_{T}=L_{R}=0 \mathrm{~dB}$. We use $5.8 \mathrm{~dB}$ as margin for temporal fading ( $95 \%$ temporal availability), based on K-factors varying from $-12 \mathrm{~dB}$ to $-6 \mathrm{~dB}$, as proposed in [15] for large office environments. We consider a coverage percentage of $90 \%$ to determine $\mathrm{M}_{\mathrm{S}}$ [16].

In the evaluation, we take into account the maximum allowed value for the EIRP (Equivalent Isotropically Radiated Power) in Europe. This is $20 \mathrm{dBm}$ in the $2.4 \mathrm{GHz}$ band. In the $5 \mathrm{GHz}$ band, this is $23 \mathrm{dBm}$ up to channel 64 (further referred to as ' $5.2 \mathrm{GHz}$ ' band) and $30 \mathrm{dBm}$ from channel 100 (further referred to as ' $5.5 \mathrm{GHz}$ ' band) [17] [18]. Channels 65 to 99 are not used in $802.11 \mathrm{n}$.

\section{B. Results and discussion}

These calculations allow to make an evaluation as shown in Tables IV and V. Both tables apply to $2.4 \mathrm{GHz}$, SISO, $20 \mathrm{MHz}$ bandwidth and $30 \mathrm{~m}$ fixed range (unless otherwise mentioned in the tables). The results in Table IV are obtained using the TGn model for environment ' $\mathrm{E}$ ' ('Large office') as well as 'F' ('Large space (indoors - outdoors)'). The results in Table V are calculated with our proposed PL model, considering two different Tx positions. 
TABLE IV

THE EFFECT OF DIFFERENT LINK PARAMETERS ON \#AP, TOTAL RADIATED POWER CONSUMPTION (P) (EXPRESSED BY A

MULTIPLICATION FACTOR) AND TP MAX , ASSUMING A FIXED RANGE OF $30 \mathrm{M}$ (UNLESS OTHERWISE MENTIONED). THIS CALCULATION IS BASED ON THE TGN MODEL FOR ENVIRONMENT 'E' AND 'F' RESPECTIVELY. THIS MAY GIVE DIFFERENT VALUES, WHICH IS INDICATED BY (1).

\begin{tabular}{|c|c|c|c|c|}
\cline { 3 - 5 } \multicolumn{1}{c|}{} & \#AP & $\mathrm{P}[\mathrm{mW}]$ & TP $_{\max }[\mathrm{Mbps}]$ \\
\hline \multirow{2}{*}{ frequency } & $2.4 \rightarrow 5.2 \mathrm{GHz}$ & $=$ & $\times 4.7$ & $65 \rightarrow 39-58.5^{(1)}$ \\
& $2.4 \rightarrow 5.5 \mathrm{GHz}$ & $=$ & $\times 5.3$ & $65 \rightarrow 65$ \\
\hline configuration & SISO $\rightarrow$ MIMO $2 \times 2: 1$ & $=$ & $\times 0.25$ & $65 \rightarrow 65$ \\
& SISO $\rightarrow$ MIMO $2 \times 2: 2$ & $=$ & $=$ & $65 \rightarrow 130$ \\
\hline bandwidth & $20 \rightarrow 40 \mathrm{MHz}$ & $=$ & $\times 2.0$ & $65 \rightarrow 81-135^{(1)}$ \\
\hline range & $30 \rightarrow 15 \mathrm{~m}$ & $\times 4$ & $\times 0.35-0.41^{(1)}$ & $65 \rightarrow 65$ \\
\hline
\end{tabular}

TABLE V

THE EFFECT OF DIFFERENT LINK PARAMETERS ON \#AP, TOTAL RADIATED POWER CONSUMPTION (P) (EXPRESSED BY A MULTIPLICATION FACTOR) AND TP $\mathrm{TAX}_{\mathrm{MA}}$, ASSUMING A FIXED RANGE OF $30 \mathrm{M}$ (UNLESS OTHERWISE MENTIONED). THIS CALCULATION IS BASED ON OUR PL MODEL FOR THE TWO TX POSITIONS: IN FRONT AND AT THE SIDE RESPECTIVELY. THIS MAY GIVE DIFFERENT VALUES, WHICH IS INDICATED BY (1).

\begin{tabular}{|c|c|c|c|c|}
\cline { 3 - 5 } \multicolumn{2}{c|}{} & \#AP & $\mathrm{P}[\mathrm{mW}]$ & $\mathrm{TP}_{\max }[\mathrm{Mbps}]$ \\
\hline \multirow{2}{*}{ frequency } & $2.4 \rightarrow 5.2 \mathrm{GHz}$ & $=$ & $\times 2.7-3.3^{(1)}$ & $65 \rightarrow 65$ \\
& $2.4 \rightarrow 5.5 \mathrm{GHz}$ & $=$ & $\times 2.7-3.3^{(1)}$ & $65 \rightarrow 65$ \\
\hline configuration & $\mathrm{SISO} \rightarrow \mathrm{MIMO} 2 \times 2: 1$ & $=$ & $\times 0.25$ & $65 \rightarrow 65$ \\
& $\mathrm{SISO} \rightarrow \mathrm{MIMO} 2 \times 2: 2$ & $=$ & $=$ & $65 \rightarrow 130$ \\
\hline bandwidth & $20 \rightarrow 40 \mathrm{MHz}$ & $=$ & $\times 2.0$ & $65 \rightarrow 135$ \\
\hline range & $30 \rightarrow 15 \mathrm{~m}$ & $\times 4$ & $\times 1.5-1.2^{(1)}$ & $65 \rightarrow 65$ \\
\hline
\end{tabular}

1) Effect of the frequency: At a higher frequency $(5.2 / 5.5$ vs $2.4 \mathrm{GHz})$, the required total radiated power consumption $\mathrm{P}[\mathrm{mW}]$ increases with a factor 3 to 5 (for the same modulation type) (see Tables IV and V). Indeed, at a higher frequency, there is a higher $\mathrm{PL}$, which requires a higher transmit power $\mathrm{P}_{\mathrm{T}}$ to maintain the same range. The PL increases with frequency $f$, due to the interaction between electromagnetic field and antenna (linear-scaled PL proportional to $\mathrm{f}^{2}$ ), which has a stronger effect than the interaction between electromagnetic field and environment (see Section III-B). The latter explains the different effect on the radiated power consumption $\mathrm{P}$ according to the TGn model (multiplication factor 5 approx) and our PL model (multiplication factor 3 approx). Note that our PL model, which has been determined at $5.4 \mathrm{GHz}$, is used for the prediction at 5.2 and $5.5 \mathrm{GHz}$. This is a good approximation, assuming that the (linear-scaled) PL varies roughly as $\mathrm{f}^{2}$ (see Section III-B).

A higher frequency $(5.2 / 5.5 \mathrm{vs} 2.4 \mathrm{GHz})$ has two different effects on the maximum possible throughput $\mathrm{TP}_{\max }$. Firstly, due to the higher PL at higher frequencies, a higher transmit power $\mathrm{P}_{\mathrm{T}}$ is required for a certain MCS. When this transmit power exceeds the maximum allowed radiated power, this MCS is no longer possible and $\mathrm{TP}_{\max }$ decreases. Secondly, the maximum allowed radiated power is higher at a higher frequency $(20,23$ and $30 \mathrm{dBm}$ EIRP at 2.4, 5.2 and 5.5 GHz resp.), which can have an increasing effect on $\mathrm{TP}_{\max }$. The total effect on $\mathrm{TP}_{\max }$ can be 
decreasing or increasing. According to the TGn model (see Table IV), $\mathrm{TP}_{\max }$ at $5.2 \mathrm{GHz}$ is lower than at $2.4 \mathrm{GHz}$. This decrease of $\mathrm{TP}_{\max }$ is stronger for environment ' $\mathrm{E}$ ' than for ' $\mathrm{F}$ ' due to the higher PL (see Section III-B). Compared to $2.4 \mathrm{GHz}, \mathrm{TP}_{\max }$ keeps the maximum value of $65 \mathrm{Mbps}$ at $5.5 \mathrm{GHz}$. This is due to the EIRP limit of $30 \mathrm{dBm}$, which is higher than the $23 \mathrm{dBm}$ limit at $5.2 \mathrm{GHz}$. According to our PL model (see Table V), $\mathrm{TP}_{\max }$ keeps the maximum value of $65 \mathrm{Mbps}$ at both 5.2 and $5.5 \mathrm{GHz}$. This can be explained by the lower PL, according to our PL model.

2) Effect of configuration and bandwidth: According to the TGn model and our PL model, the total radiated power consumption $\mathrm{P}$ [mW] decreases with a factor 4 for MIMO $2 \times 2: 1$ (vs SISO) (see Tables IV and V). P remains unchanged for MIMO $2 \times 2: 2$ (vs SISO) and increases with a factor 2 for $40 \mathrm{MHz}$ bandwidth (vs $20 \mathrm{MHz}$ ). Indeed, from (7) and (9), and taking into account that the number of access points (\#AP) is fixed, it is clear that the increase (decrease) of $P[\mathrm{dBm}]$ equals the increase (decrease) of the receiver sensitivity $P_{R}[\mathrm{dBm}]$, irrespective of the PL model. This can be easily understood: when the sensitivity [mW] increases with a certain factor, the transmit power $\mathrm{P}_{\mathrm{T}}[\mathrm{mW}]$ should increase with the same factor to keep the range unchanged.

Compared to SISO, MIMO $2 \times 2: 1$ can generally result in an increased $\mathrm{TP}_{\max }$, when for SISO the transmit power $\mathrm{P}_{\mathrm{T}}$, required to obtain the fixed range, exceeds the maximum allowed radiated power. Indeed, the lower sensitivity $\mathrm{P}_{\mathrm{R}}$ of MIMO $2 \times 2: 1$ allows to achieve the fixed range with a lower transmit power $\mathrm{P}_{\mathrm{T}}$. In particular, we see in Tables IV and V that the maximum data rate of $65 \mathrm{Mbps}$ remains unchanged. Compared to SISO, $\mathrm{TP}_{\max }$ doubles for MIMO $2 \times 2: 2$, due to the two spatial streams (multiplexing).

A higher bandwidth (40 vs $20 \mathrm{MHz}$ ) has two different effects on the maximum possible throughput $\mathrm{TP}_{\max }$. Firstly, $\mathrm{TP}_{\max }$ increases approximately with a factor 2 (e.g. from 65 to $135 \mathrm{Mbps}$ ). Secondly, the higher receiver sensitivity $\mathrm{P}_{\mathrm{R}}$ can have a decreasing effect on $\mathrm{TP}_{\max }$ (as explained before). The latter effect occurs according the TGn model (environment 'E') (see Table IV), while this effect does not occur according to our PL model (see Table V). This different prediction can be explained by the lower PL according to our PL model.

3) Effect of the range: For a lower fixed range (15 vs $30 \mathrm{~m})$, the required number of access points increases of course (with a factor 4 , see (8)). The total radiated power P decreases (with a factor 2.4 to 2.9) according to the TGn model, while P increases (with a factor 1.2 to 1.5) according to our PL model. This can be explained as follows. From (7), it is clear that the transmit power $\mathrm{P}_{\mathrm{T}}[\mathrm{mW}]$ is proportional to $\mathrm{R}^{\mathrm{n}}$, where $\mathrm{R}$ is the range $[\mathrm{m}]$ and $n$ the PL exponent. Consequently, it follows from (8) and (9) that $P$ is proportional to $R^{n-2}$. A higher fixed range $\mathrm{R}$ has an increasing (decreasing) effect on $\mathrm{P}$, when the PL exponent $\mathrm{n}$ is higher (lower) than 2 . According to the TGn model, the PL exponent is 2 to 3.5, which indeed results in an increasing effect on P (for increasing range). According to our PL model, the PL exponent is lower than 2 (see Table I), which results in a decreasing effect on $P$ (for increasing range).

A lower fixed range can generally result in an increased maximum possible throughput $\mathrm{TP}_{\max }$, when the transmit power $\mathrm{P}_{\mathrm{T}}$, required to obtain the fixed range, exceeds the maximum allowed radiated power. In particular, the maximum data rate of $65 \mathrm{Mbps}$ remains unchanged (see Tables IV and V).

The mentioned contradiction between the two PL models on the power consumption is further illustrated in Figs. 7 and 8, which show the required total radiated power consumption $\mathrm{P}$ as a function of the transmit power $\mathrm{P}_{\mathrm{T}}$ according to the TGn model (environment 'F') and our PL model (Tx position in front) respectively. The calculation is done for 2.4 and $5.5 \mathrm{GHz}$, SISO, $20 \mathrm{MHz}$ bandwidth, modulation scheme 64-QAM 5/6 (65 Mbps) and an area of $10,000 \mathrm{~m}^{2}$ (large conference room). The calculation is based on (7) - (9), where the number of access points 
(\#AP) has been rounded up to an integer. It can be seen that there is indeed an overall increasing (or stagnating) trend of $\mathrm{P}$ vs $\mathrm{P}_{\mathrm{T}}$ according to the TGn model (see Fig. 7) and an overall decreasing trend according to our PL model (see Fig. 8). This can be understood: since $\mathrm{P}_{\mathrm{T}}[\mathrm{mW}]$ is proportional to $\mathrm{R}^{\mathrm{n}}$, it is clear from (8) and (9) that $\mathrm{P}$ is proportional to $\mathrm{P}_{\mathrm{T}}^{-2 / \mathrm{n}+1}$. Consequently, the overall trend is increasing (or stagnating) for a PL exponent higher than (or equal to) 2 (TGn model) and decreasing for a PL exponent lower than 2 (our PL model). The effect of rounding up the number of access points (i.e. the tooth profile in Figs. 7 and 8) can be explained as follows. First, $\mathrm{P}[\mathrm{mW}]$ increases linearly with increasing $\mathrm{P}_{\mathrm{T}}[\mathrm{mW}]$, because the number of access points remains constant. Then, when the range has been increased sufficiently so that one access point can be left out, $\mathrm{P}$ falls back to the $\mathrm{P}_{\mathrm{T}}^{-2 / \mathrm{n}+1}$ relationship (see Figs. 7 and 8). This effect is negligible when the number of access points is sufficiently high (i.e. for low $\mathrm{P}_{\mathrm{T}}$ ) (see Figs. 7 and 8). Note that when only one access point is required (i.e. for sufficiently high $\mathrm{P}_{\mathrm{T}}$ ), $\mathrm{P}$ $[\mathrm{mW}]$ increases linearly with $\mathrm{P}_{\mathrm{T}}[\mathrm{mW}]$ (see e.g. Fig. 8).

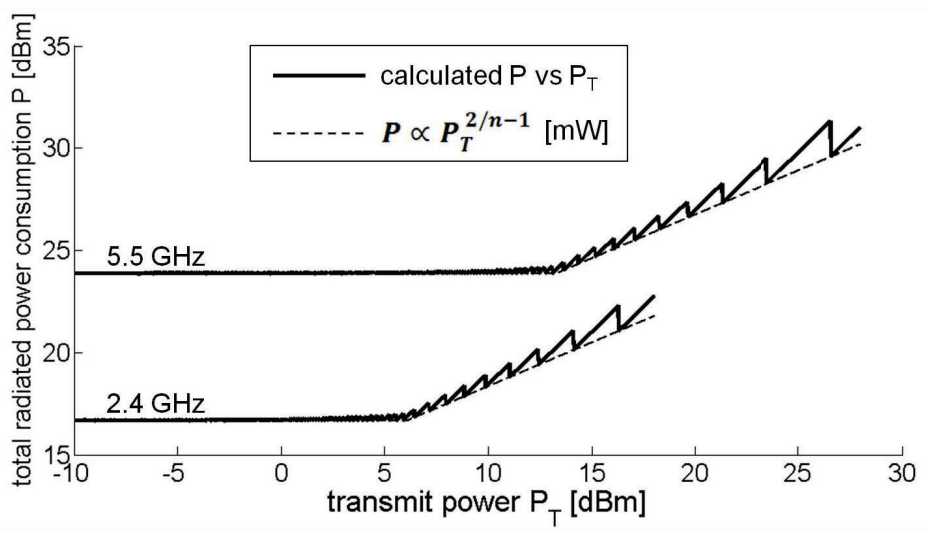

Fig. 7. Total radiated power consumption $P$ (solid line) as a function of the transmit power $\mathrm{P}_{\mathrm{T}}$. The calculation is based on the TGn model. An overall stagnating or increasing trend of $\mathrm{P} v \mathrm{vs} \mathrm{P}_{\mathrm{T}}$ is found. The dashed line corresponds to $\mathrm{P}$, calculated without rounding up the number of access points (\#AP) in (8) and indicates the overall trend $\left(\mathrm{P}[\mathrm{mW}]\right.$ proportional to $\left.\mathrm{P}_{\mathrm{T}}^{2 / \mathrm{n}-1}\right)$.

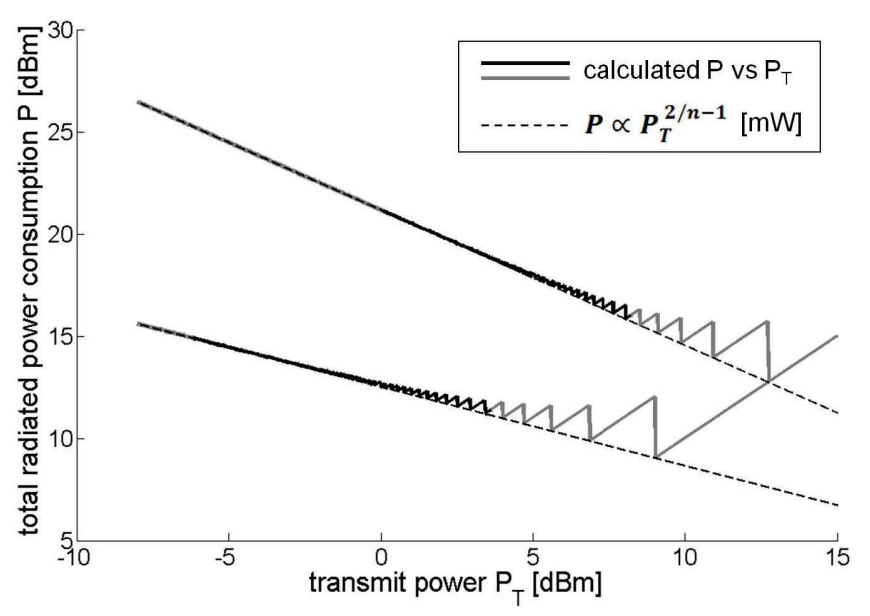

Fig. 8. Total radiated power consumption $\mathrm{P}$ (solid line) as a function of the transmit power $\mathrm{P}_{\mathrm{T}}$. The calculation is based on our PL model. An overall decreasing relation $\mathrm{P}$ vs $\mathrm{P}_{\mathrm{T}}$ is found. The dashed line corresponds to $\mathrm{P}$, calculated without rounding up the number of access points (\#AP) in (8) and indicates the overall trend ( $\mathrm{P}[\mathrm{mW}]$ proportional to $\mathrm{P}_{\mathrm{T}}^{2 / \mathrm{n}-1}$ ). The grey solid line indicates that the PL model is out of the region where the PL model could be experimentally determined. 


\section{Summary}

Finally, this evaluation can be summarized as follows. At a higher frequency (5.2/5.5 vs $2.4 \mathrm{GHz})$, a higher total radiated power $\mathrm{P}$ is required. The effect on the maximum possible throughput $\mathrm{TP}_{\max }$ can be decreasing or increasing, due to a higher EIRP limit at higher frequencies. A MIMO $2 \times 2$ configuration has only advantages: a lower $\mathrm{P}$ and a possibly higher $\mathrm{TP}_{\max }$ when using MIMO $2 \times 2: 1$, a doubled $\mathrm{TP}_{\max }$ and an unchanged $\mathrm{P}$ when using MIMO $2 \times 2: 2$. A higher bandwidth $(40$ vs $20 \mathrm{MHz}$ ) requires a higher $\mathrm{P}$ and can have an additional decreasing effect on the doubled $\mathrm{TP}_{\max }$. A higher fixed range requires of course a lower number of access points, but can have a decreasing effect on $\mathrm{TP}_{\max }$. The effect on $\mathrm{P}$ can be decreasing or increasing, depending on the PL exponent.

The predictions of the TGn model and our PL model differ essentially in two aspects. Firstly, the TGn model predicts limitations on the maximum possible throughput $\mathrm{TP}_{\max }$ at a fixed range of $30 \mathrm{~m}$ (e.g. at $5.2 \mathrm{GHz}$ or for $40 \mathrm{MHz}$ bandwidth, see Table IV), while our PL model does not (see Table V). This is due to the lower PL according to our PL model. Secondly, the TGn model predicts that a higher fixed range requires a higher (or unchanged) total radiated power P, while our PL model predicts a lower required P. This is due to a PL exponent higher than (or equal to) 2 according to the TGn model and a PL exponent lower than 2 according to our PL model. We conclude that our PL model is significantly better than the TGn model for the prediction of the range, (radiated) power consumption and throughput.

\section{CONCLUSiOnS}

We determined a PL model for 802.11n in large conference rooms, based on PL measurements. The PL could be described accurately by a one-slope model. In the absence of humans, PL exponents varying from 1.2 to 1.7 were found, which is lower than the PL exponents, proposed by the TGn channel model (2 to 3.5). Based on PL measurements in the presence of humans, the PL exponent increases towards 2.

The effect of frequency $(2.4 / 5 \mathrm{GHz})$, configuration (SISO vs MIMO $2 \times 2$ ), bandwidth $(20 \mathrm{vs} 40 \mathrm{MHz}$ ) and transmit power on the required number of access points, total radiated power consumption $\mathrm{P}$, and maximum (physical) throughput $\left(\mathrm{TP}_{\max }\right)$ has been investigated. This has been done by link budget calculation, based on our proposed PL model as well as the TGn channel model.

The predictions of the two PL models differ essentially in two aspects. Firstly, the TGn model predicts limitations on the maximum throughput $\mathrm{TP}_{\max }$ at a (fixed) range of $30 \mathrm{~m}$, while our proposed PL model does not. This is due to a lower PL according to our PL model. Secondly, according to the TGn model, a higher (fixed) range requires a higher (or unchanged) radiated power consumption P, while our PL model predicts a lower required P. This is due to a PL exponent higher than (or equal to) 2 according to the TGn model and a PL exponent lower than 2 according to our PL model. We conclude that our PL model is significantly better than the TGn model for the prediction of the range, (radiated) power consumption and throughput.

Future research will consist of MIMO channel modeling in large conference rooms.

\section{ACKNOWLEDGMENT}

This work was supported by the OMUS (Optimizing Multimedia Service Delivery) project, co-funded by the IBBT (Interdisciplinary institute for BroadBand Technology), a research institute founded by the Flemish Government in 2004, and the involved companies and institutions. W. Joseph is a postdoctoral fellow of the FWO-Flanders. 


\section{REFERENCES}

[1] IEEE Std 802.11n ${ }^{T M}-2009$ amendment 5 to part 11: Wireless LAN Medium Access Control (MAC) and Physical Layer (PHY) specifications: enhancements for higher throughput, IEEE Std., Oct 2009.

[2] J. Tarng and T. Liu, "Effective models in evaluating radio coverage on single floors of multifloor buildings," Vehicular Technology, IEEE Transactions on, vol. 48, no. 3, pp. 782-9, 1999.

[3] V. Erceg et al., "TGn channel models," IEEE, Tech. Rep., May 2004.

[4] N. Papadakis, A. Economou, J. Fotinopoulou, and P. Constantinou, "Radio propagation measurements and modeling of indoor channels at $1800 \mathrm{MHz}$, Wireless Personal Communications, vol. 9, no. 2, pp. 95-111, 1999.

[5] S. Phaiboon, P. Phokharatkul, and S. Somkuarnpanit, "New upper and lower bounds line of sight path loss model for mobile propagation in buildings," AEU - International Journal of Electronics and Communications, vol. 62, no. 3, pp. 207-215, 2008.

[6] T. R. Liu and J. H. Tarng, "Modeling and measurement of 2.44-GHz radio out-of-sight propagation on single floors," Microwave and Optical Technology Letters, vol. 14, no. 1, pp. 56-59, 1997.

[7] K. W. Cheung, J.-M. Sau, and R. Murch, "A new empirical model for indoor propagation prediction," Vehicular Technology, IEEE Transactions on, vol. 47, no. 3, pp. 996-1001, 1998.

[8] C.-M. Chen, C.-C. Chiu, C.-H. Chen, and Y.-C. Chen, "A novel propagation-prediction model for small rooms with metallic furniture," Microwave and Optical Technology Letters, vol. 44, no. 3, pp. 281-4, 2005.

[9] E. Tanghe et al., "The industrial indoor channel: large-scale and temporal fading at 900, 2400, and 5200 MHz," IEEE Transactions on Wireless Communications, vol. 7, no. 7, pp. 2740-51, 2008.

[10] _ - "Statistical validation of WLAN range calculated with propagation models for industrial environments by chipset-level received signal strength measurements," IET Science, Measurement and Technology, vol. 3, no. 3, pp. 244-255, 2009.

[11] D. De Zutter and F. Olyslager, Applied electromagnetics. Ghent, Belgium:Ghent University, 2005.

[12] J. Dabin, A. Haimovich, and H. Grebel, "A statistical ultra-wideband indoor channel model and the effects of antenna directivity on path loss and multipath propagation," IEEE Journal on Selected Areas in Communications, vol. 24, no. 4, pp. 752-8, 2006.

[13] Wikipedia. [Online]. Available: en.wikipedia.org/wiki/IEEE_802.11n

[14] A. Sibille, "Time-domain diversity in ultra-wideband MIMO communications," EURASIP Journal on Advances in Signal Processing, vol. 2005, no. 3, pp. 316-327, 2005.

[15] R. J. C. Bultitude, "Measurement, characterization and modeling of indoor 800/900 MHz radio channels for digital communications," IEEE Commun. Mag., vol. 25, no. 6, pp. 5-12, 1987.

[16] S. Saunders and A. Aragón-Zavala, Antennas and propagation for wireless communication systems, 2nd ed. John Wiley \& Sons Ltd, 2007.

[17] Wikipedia. [Online]. Available: en.wikipedia.org/wiki/List_of_WLAN_channels

[18] Belgian Institute for Postal services and Telecommunications. [Online]. Available: http://www.bipt.be

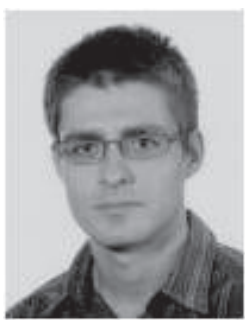

Frederic Heereman was born in 1981 in Belgium. He graduated as Master of Science in Engineering Physics at Ghent University (Belgium) in 2008. Since July 2010, he is research assistant at IBBT-UGent/INTEC (Interdisciplinary institute for BroadBand Technology / Department of Information Technology) of the same university. His scientific work is focused on indoor propagation aspects of the wireless physical layer. 


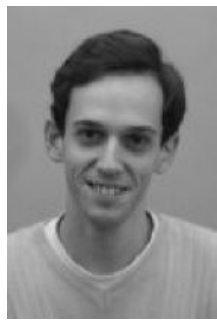

Wout Joseph was born in Ostend, Belgium on October 21, 1977. He received the M. Sc. degree in electrical engineering from Ghent University (Belgium) in July 2000. From September 2000 to March 2005, he was a research assistant at the Department of Information Technology (INTEC) of the same university. During this period, his scientific work was focused on electromagnetic exposure assessment. This work led to a Ph. D. degree in March 2005. Since April 2005, he is postdoctoral researcher for IBBT-Ugent/INTEC. Since October 2007, he is a postdoctoral fellow of the FWO-V (Research Foundation - Flanders). His professional interests are electromagnetic field exposure assessment, propagation for wireless communication systems, antennas and calibration. Furthermore, he specializes in wireless performance analysis and quality of experience.

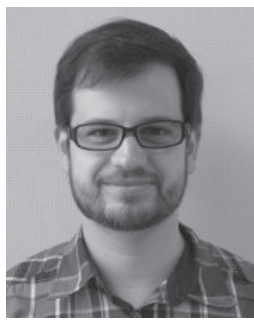

Emmeric Tanghe (Ph. D., M. Sc. Eng.) was born in Tielt, Belgium on August 31, 1982. He received the M. Sc. degree in electrical engineering from Ghent University (Belgium) in July 2005. From September 2005 to May 2011, he was a research assistant at IBBT-Ugent/INTEC (Interdisciplinary institute for BroadBand Technology / Department of Information Technology) of the same university. His scientific work focused on the modeling of indoor and outdoor propagation through field measurements. This work led to a Ph. D. degree in May 2011. Since May 2011, he is postdoctoral researcher at same institution continuing his work in propagation modeling.

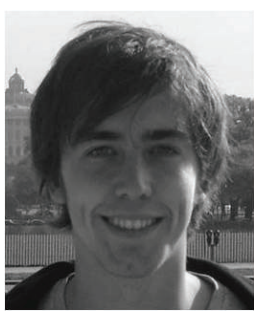

David Plets was born in 1983 in Belgium. In 2006, he obtained a Master in Electrotechnical Engineering, with ICT as main subject. Currently, he is a member of the WiCa group (Department of Information Technology - INTEC, Ghent University). In 2011, he obtained his $\mathrm{PhD}$ with a dissertation on the characterization and optimization of the coverage of wireless networks. His research interests include performance and optimization of, and propagation in DVB-H and WLANs.

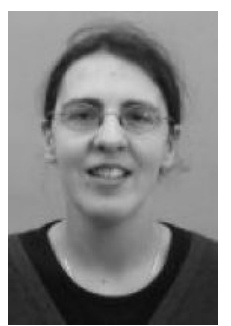

Leen Verloock was born in Eeklo, Belgium, on November 15, 1979. She received the degree of Master in electronics engineering from Katholieke Hogeschool Ghent (Belgium) in 2001. She then joined the Department of Information Technology (INTEC) of Ghent University where she is currently working as a technical and research assistant in the Wireless and Cable Research group. She is working on measuring and modeling the propagation of electromagnetics fields around wireless systems. She is also doing measurements of electromagnetic fields around base stations in order to check their compliance with the exposure limits. 


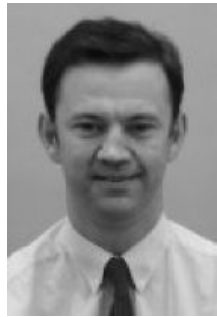

Luc Martens was born in Ghent, Belgium on May 14, 1963. He received the M. Sc. degree in electrical engineering from Ghent University (Belgium) in July 1986. From September 1986 to December 1990 he was a research assistant at the Department of Information Technology (INTEC) of the same university. During this period, his scientific work was focused on the physical aspects of hyperthermic cancer therapy. This work led to a Ph. D. degree in December 1990. Since January 1991, he is a member of the permanent staff of the Interuniversity MicroElectronics Centre (IMEC), Ghent, and is responsible for the research on experimental characterization of the physical layer of telecommunication systems at INTEC. His group also studies topics related to the health effects of wireless communication devices. Since April 1993 he is Professor in electrical applications of electromagnetism at Ghent University. 